\title{
AN ECONOMIC MODEL OF STUDENT LEARNING
}

\author{
ANDREW BARKLEY* \\ Department of Agricultural Economics, Kansas State University, Manhattan, Kansas \\ BRIAN K. COFFEY \\ Department of Agricultural Economics, Kansas State University, Manhattan, Kansas
}

\begin{abstract}
We develop a dynamic economic model based on contemporary understanding of learning and motivation. The model and resulting comparative static analysis show the instructor's role in providing a productive learning environment. Instructors should set the complexity of course assignments and assessments to be consistent with student preparedness. Learning is enhanced when the rate of change in challenge is based on the rate of change of how rapidly students are learning. Instructors steer students toward a productive trajectory with tasks that cause students to work hard and by consistently providing feedback and fostering a culture of learning.
\end{abstract}

Keywords. Dynamic learning model, flow motivation theory, student learning

JEL Classifications. A22, C61, D03

\section{Introduction}

Economic models have been used extensively to illuminate several areas of teaching and learning. Becker and Baumol (1995) offer a collection of such contributions, along with a history of the use of economic modeling in the scholarship of teaching and learning. Economic research often involves analyzing messy, observed data (as opposed to data from controlled, randomized experiments) and identifying cause and effect relationships (Baumol and Becker, 1995). Logistical and ethical concerns limit truly randomized experiments in education, meaning that researchers rely on ex post facto design (Becker, 1983b), making econometric tools of economists well suited for evaluating these data. The majority of economic modeling related to the scholarship of teaching and learning has used data of student outcomes to identify benefits of alternative teaching methods or learning environments. There have been many useful

\footnotetext{
*Corresponding author's e-mail: barkley@ksu.edu

We would like to express our sincere appreciation to Professor Norman Obst, who provided great insight, and editor Michael Reed for his outstanding support and suggestions.
} 
findings from such endeavors even though there are limitations and challenges with existing studies (Becker, 2004).

A common approach to evaluating student outcomes and teaching effectiveness has been to specify a production function (Monk, 1989) using data collected from classrooms (e.g., Bacdayan, 1997; Becker, 1983a; Charkins et al., 1985; Dahlgran, 1990; Flyer and Rosen, 1996; Manahan, 1983). A far less explored area of research, which economic tools are equally well equipped to address, is applying conceptual economic models to teaching and learning (Lima, 1981; Wetzstein and Broder, 1985). Lima (1981, p. 1056) lamented that "it is virtually impossible to discover attempts to derive hypotheses about determinants of good teaching from explicit models of behavior." There are exceptions. Becker (1983a) proposed an inventory model based on money demand modeling in macroeconomics. Bacdayan (1997) expanded the production function approach to address differences in the learning process across individuals. These studies, however, follow the convention of equating student outcomes with learning. Lima (1981) and Wetzstein and Broder (1985) propose behavioral models and draw conclusions. Also rare in economic educational models is consideration of contemporary psychology and education research (Rauh and Seccia, 2006). In fact, understanding the learning process in general remains an area of research that is rich with opportunity.

In 1995, Barr and Tagg challenged university teachers and administrators to transform academic institutions from "instruction-centered" to "learningcentered" campuses. Since then, a great deal of time and attention has been given to improving teaching and learning at colleges and universities. New methods of instruction have been developed and evaluated, and the assessment of student learning outcomes has proliferated. In spite of this enhanced level of attention to pedagogy, our understanding of how students learn remains weak (Smith, 1999; Stage et al., 1998). The research presented here fills these voids by developing a conceptual economic model of the learning process that is consistent with psychological theories of learning and then drawing implications from the model to guide teaching practices.

The objective of this study is to propose and specify a dynamic mathematical model of learning that considers students' psychological response to instruction, the complexity of instruction, the rate of increasing difficulty of instruction, and student-instructor interaction. The model demonstrates the importance of calibrating how rapidly the complexity of material is increased with the preparedness of students. Properly balancing the rate of increase of the challenge presented to students with their readiness to meet the challenge effectively motivates students and allows them to achieve a steady-state condition of learning. Implications are derived for a variety of courses, including small seminars, field-type courses, large required courses, and graduate courses.

The application of the theoretical model to individual students with heterogeneous knowledge and ability levels is also explored. A case for pragmatic 
implementation of the model as a dynamic classroom is made. Further research based on the theoretical model presented here is also discussed. The crux of the theoretical model is for teachers to pay careful attention to student learning and adjust the assignments, expectations, and course assessments to better match where students are at the time. Brookfield (2015) concludes, "The most important knowledge that skillful teachers need to do good work is a constant awareness of how students are experiencing their learning and perceiving teachers' actions" (p. 15). This quote synthesizes and summarizes the case for a dynamic classroom based on the theoretical model of dynamic learning, as detailed in what follows.

\section{Flow Theory of Motivation}

The psychology of motivation suggests that work is most productive and people are most content while in a state of flow. The concept of flow was originally defined by Csikszentmihalyi (1975) as a state of deep focus that occurs when people engage in challenging tasks that demand intense concentration and commitment. Flow is considered to occur when a person's skill level is perfectly balanced with the level of challenge for a task (Shernoff et al., 2003) that has clear goals and provides immediate feedback. Flow experience is an enjoyable, productive experience. Csikszentmihalyi (2003) states: "Our research shows that teenagers who are in the flow more often develop more productive habits: Not only are they much happier and more optimistic, and have higher self-esteem, but they study more, are involved in active leisure more often, and spend more time with friends - a finding that is independent of income, parental education, and social status" (p. 69). The foundation of flow theory is that enjoyable and productive experiences balance an individual's capacity (or skill set) with opportunity (or challenge).

The term "challenge" forms the foundation of the model presented in this research and is broadly defined as the level of complexity of a task. Thus, challenge incorporates student learning outcomes, instructional design, and teaching strategies. This all-encompassing definition of challenge has been extensively studied in many theories of human motivation, including effectance motivation, perceived competence, flow states, and self-efficacy (Lepper et al., 1993). Lepper et al. (1993) emphasize that challenge includes the relationship between two subjective variables: the individual learner's perceived difficulty of success and the individual's estimate of his or her own ability (readiness).

An individual student ${ }^{1}$ can be considered to be "in the flow" (also called "in the zone" in sports psychology) when his or her talents are fully employed in a learning situation that is neither too stressful nor too boring. In this

1 The term "student" is used very generally here. Flow theory can be applied to an individual learning any new task or concept. Everett and Raven (2015) apply the idea to university students. 


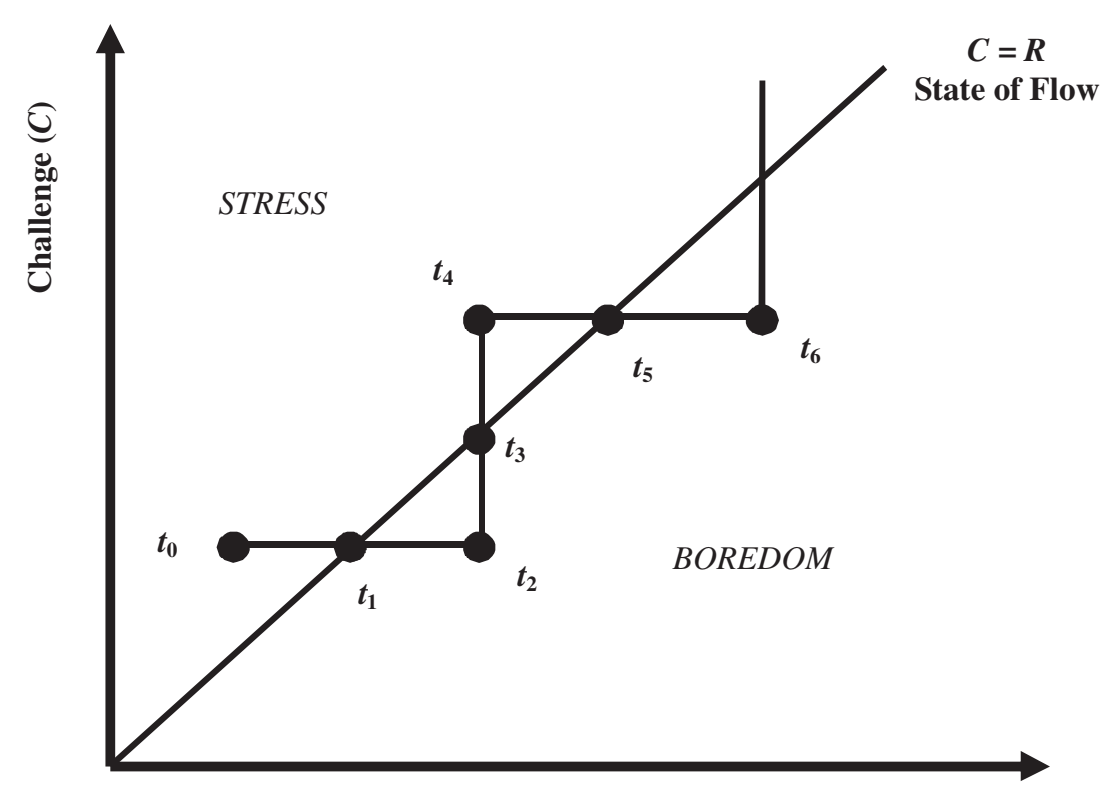

Readiness $(\boldsymbol{R})$

Figure 1. Flow Theory of Motivation (Note: This figure is based on Csikszentmihalyi's $(1975,1990,2003)$ flow theory of motivation. The 45-degree line where challenge equals readiness, $C=R$, corresponds with Csikszentmihalyi's concept of flow experience as learners master increasing complex tasks. Instructors raise the level of $C$, causing initial anxiety and motivation to perform, and, as students progress, these tasks become easier, and students drift toward the right to boredom. The instructor, in turn, again raises $C$, and the cycle of flow learning continues.)

state, the individual finds learning to be a deeply enjoyable experience. The enjoyment or euphoria from being in the flow offers not only immediate benefits of productivity, but also motivation to continue learning. We assert that college students will have the most productive learning environment when the level of complexity of course assignments is in line with each student's level of knowledge, or skill set. We define the degree of difficulty as the level of "challenge" $(C)$, and the skill set, or capability for learning a new concept or doing a task, as "readiness" $(R)$. The flow theory of learning is illustrated in Figure 1, based on Csikszentmihalyi (1990, 2003).

Figure 1 captures the relationship between challenge $(C)$ and readiness $(R)$. When challenge and readiness levels are equivalent, the student is located on the 45-degree line $(C=R)$. In the case when $C>R$, the student is located above the line, and anxiety (or stress) occurs. When $C<R$, students are located below the line and are less engaged, or bored. To the extent that there are psychological 
costs associated with both anxiety and boredom, it is optimal to be located on the $C=R$ line. ${ }^{2}$ This is true not only for the student, to avoid the costs of anxiety and boredom, but also for society as a whole. Any extended amount of time and energy spent in worry or idleness does not contribute to the attainment of societal growth and development. This description of learning is consistent with an "optimal" level of arousal (Hebb, 1955) and its connection to motivation (Apter, 1989).

The dynamic nature of flow theory is captured by a sequence of events in a learning process. Suppose that a student is learning any new and complex activity or concept. At time $t=t_{0}$ (Figure 1), the task is difficult because challenge is greater than readiness $(C>R)$, and anxiety occurs. In the short term, this anxiety can motivate the student to higher achievement (Rauh and Seccia [2006] explore this idea in the context of economic education). With experience, the task becomes easier, and readiness increases until the $C=R$ line is reached at $t=t_{1}$, when the task is mastered, and the student may experience flow, or a state of euphoria. This experience is described by Csikszentmihalyi (2003): "Think of how an athlete or a violinist feels when he is in flow: He is doing something that is objectively extremely difficult, but to him it feels almost effortless" (p. 67). Learning a new concept is often associated with this type of emotion, as seemingly random words and ideas crystallize and become understandable-the "aha" moment. ${ }^{3}$ The student's ability eventually outpaces the degree of difficulty, and the student becomes bored at time $t=t_{2}(C<R)$. The student is ready for a higher level of complexity, and the learning cycle begins anew when the challenge level is increased from $t_{2}$ to $t_{4}$.

This simple model of learning highlights a cycle of anxiety when difficult new concepts are introduced $\left(t_{0}\right)$, followed by a state of flow when expectations are met $\left(t_{1}, C=R\right)$, and finally a state of boredom when the level of student readiness outgrows the level of challenge $\left(t_{2}\right)$. According to Csikszentmihalyi (2003), "Those who are able to find the middle way weave opportunities and ability together in an enjoyable progress toward complexity" (p. 69). If the learning environment can maintain appropriately balanced levels of challenge and readiness, students can progress more rapidly and enjoyably toward higher levels of complexity, represented by the northeast direction in Figure 1. Any action or policy that minimizes time spent off of the $C=R$ line will lower

2 The 45-degree line is a result of scaling the two variables $C$ and $R$. Different scaling would result in different slopes, with the qualitative results unchanged. The linear relationship is a result of assuming constant marginal challenge. A more general, realistic model could include diminishing marginal challenge. Here, we assume constant marginal challenge in the relevant range of challenge and readiness; thus the true relationship is approximated with linearity.

3 Psychologists have uncovered the nature of how the brain discovers new insights. Bowden et al. (2005, p. 325) found that insight is achieved in the right hemisphere of the brain, which "engages in relatively coarse semantic coding, and is therefore more likely to maintain diffuse activation of alternative meanings, distant associations and solution-relevant concepts." 
costs and enhance welfare for both the individual student and society as a whole. The role of the teacher is to attempt to move the student to higher levels of task complexity by properly balancing challenge and complexity of course requirements with student readiness levels in a dynamic framework.

\section{A Simple Model of Learning: The Learning Adjustment Mechanism}

The mechanics of the learning adjustment mechanism are outlined in this section, and then expanded to a more complete dynamic model in the next section. Let $K$ be the total level of knowledge (a stock variable) attained by a student over a given time interval, $t=t_{0} \ldots T$. The rate of learning is $k$ (a flow variable ${ }^{4}$ ), equal to the percentage rate of change in knowledge, as in the learning adjustment equation (1), where both stress and boredom have a negative impact on the learning rate: ${ }^{5}$

$$
k=(\partial K / \partial t) / K=k_{\max }-\beta[(R-C) / C]^{2}=k_{\max }-\beta[(R / C)-1]^{2},
$$

where $k_{\max }$ is defined to be a maximum level of the learning rate that occurs when $R=C$. The parameter $\beta$ is a positive constant $(\beta>0)$, and the variables $R$ and $C$ maintain their previous definitions. The term $[(R-C) / C]$ represents the proportion of a student's ability, or readiness, that is left unchallenged. The greater the proportion of untapped student ability (i.e., boredom), the slower the rate of learning. Similarly, too much challenge creates stress and also reduces the learning rate. More specifically, whenever $C$ diverges from $R$, the learning rate $(k)$ decreases from the maximum rate of learning $\left(k_{\max }\right)$. When challenge is greater than student readiness $(C>R)$, or when a student's level of readiness to learn is greater than the level of challenge $(R>C)$, the rate of learning is smaller than the maximum learning rate $\left(k_{\max }\right)$. Restated, a balanced level of challenge and readiness $(R / C=1)$ results in a maximum learning rate $\left(k_{\max }\right)$.

To allow for the possibility that the optimal learning rate takes place at a ratio of readiness to challenge that differs from 1 , the parameter $S$ is included in equation (2). For example, Nilson (2016) emphasizes “desirable difficulties," or the idea that "people can remember what they have learned longer when they have to work harder to learn it" (p. 5). The parameter $S$ reflects the level of $R / C$ that causes the learning rate $(k)$ to be at a maximum $\left(k_{\max }\right)$.

$$
k=k_{\max }-\beta[(R / C)-S]^{2}
$$

4 The term "flow" is used here in the mathematical sense to mean rate of change of a stock variable. The fact that Csikszentmihalyi $(1990,2003)$ chose the same term to describe the optimal learning pathway is coincidental.

5 Note that equation (1) assumes that the learning process takes place within the relevant range, where $0<R<b$ and $0<C<h$, where $h$ is a positive constant. This assumption provides for a finite level of learning. 


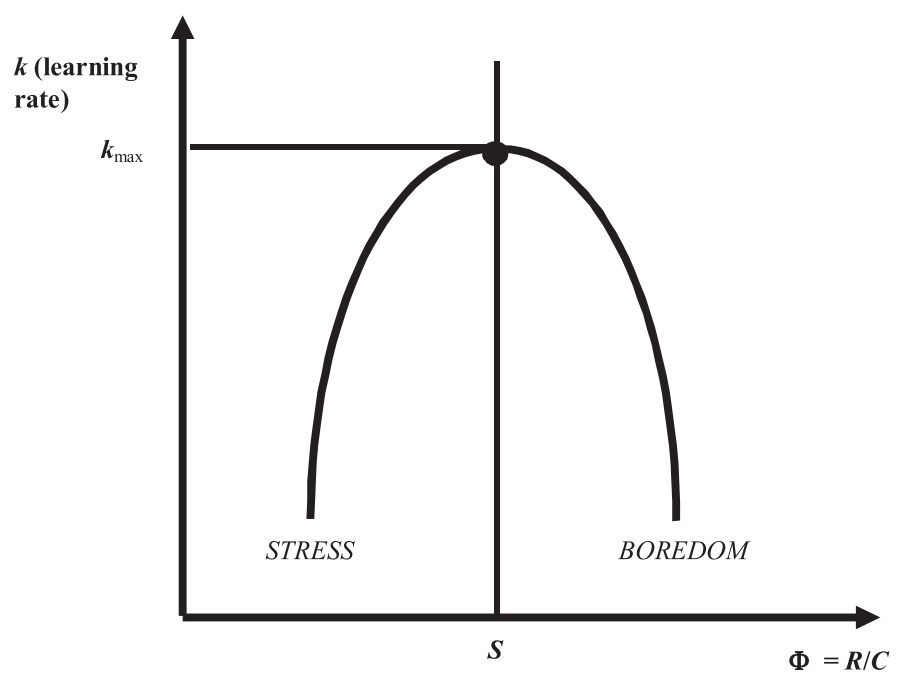

Figure 2. A Simple Model of Learning: The Learning Adjustment Mechanism (Note: The term $k_{\max }$ is the maximum learning rate; $\Phi$ is the readiness-to-challenge ratio, $R / C$. The variable $S$ is the equilibrium level of $\Phi$, associated with the maximum learning rate, $k_{\max }$.)

This could be attributable to optimal learning rates that occur when $C>R$, or higher rates of anxiety than readiness. To simplify further, we define $\Phi$ to be the ratio of challenge to readiness: $\Phi=R / C$.

$$
k=k_{\max }-\beta[\Phi-S]^{2}
$$

The model described here is shown in Figure 2. The first derivatives of equation (3) with respect to student readiness $(R)$ and instructor challenge $(C)$ illustrate the foundational assumptions of the simple model of the learning adjustment mechanism, as shown in equations $(4 \mathrm{a})$ and $(4 \mathrm{~b})$.

$$
\begin{aligned}
& \partial k / \partial C=-2 \beta[\Phi-S]\left(-R / C^{2}\right), \quad \partial k / \partial C>0 \text { if and only if (iff) } \Phi>S, \\
& \text { and } \partial k / \partial C<0 \text { iff } \Phi<S
\end{aligned}
$$

$$
\partial k / \partial R=-2 \beta[\Phi-S](1 / C), \quad \partial k / \partial R>0 \text { iff } \Phi<S, \text { and } \partial k / \partial R<0 \text { iff } \Phi>S
$$

The derivatives show that the learning rate depends on the relationship between $\Phi$ and $S$. This relationship corresponds directly to psychological theory of motivation and educational psychology theories of learning. Specifically, cognitive load theory (Artino, 2008) asserts that short-term memory (defined as "working memory") is very limited in both capacity and duration, whereas longterm memory is virtually limitless. The implication is that learning is slowed if 
instructional materials overwhelm a learner's limited working memory resources $(\Phi<S)$. Thus, the theory focuses on reducing all unimportant information presented to students (Artino, 2008). In the terminology of this article, properly balancing challenge with student readiness brings $\Phi$ into equality with $S$.

Student readiness is related to prior knowledge $(K)$, together with the external motivation (or drive) to learn new knowledge, represented as $M$. Thus, the motivation level can be considered to be an "efficiency coefficient" on knowledge. Motivation levels are complex and vary widely across individual students. Individual differences in motivation will be explored in greater detail later. For now, we assume an exogenous level of $M$ for a representative student. If we assume that readiness is directly proportional to knowledge, adjusted by motivation, equation (5) results, where the parameter $a$ is a constant, representing the degree to which ability is transferred into learning.

$$
\Phi=R / C=a K M / C
$$

This formulation is consistent with cognitive construction theory, which emphasizes the idea that the learner builds on preexisting knowledge to acquire new information (Loyens and Gijbels, 2008). Thus, the learner is helping herself, rather than just memorizing facts presented by the teacher. Because knowledge is increasing over time, readiness (supply) is also increasing, and challenge (demand) shifts to maintain the model equilibrium. Therefore, the simple model depicted previously does not fully capture the flow theory of learning, and a more complete dynamic flow model is described in the next section.

\section{A Flow Model of Learning}

Obst (1978) observed that current economic models of the money supply were inconsistent with real-world events, and developed a dynamic model that more closely matched real-world conditions. The base model of money supply is an inventory model. That is, it models a stock of something that changes over time. Learning can be modeled in much the same way (Becker, 1983a). That is, learning is seen as some level of knowledge that students accumulate over time. Following this intuition, we adapt Obst's (1978) dynamic model to the learning process over time, considering the previously discussed concepts of Csikszentmihalyi's (1990, 2003) flow theory of motivation. The simple model of the learning adjustment mechanism captures the major features of the flow model of learning and how the relationship between readiness and challenge affect the learning rate at a given moment in time. The simple learning adjustment mechanism described previously implies that the maximum achievable level of learning $\left(k_{\max }\right)$ is also the steadystate equilibrium level of learning that occurs when $R=C$. However, because $k_{\max }$ does not consider future learning, the model is myopic, and $k_{\max }$ in the simple model cannot be considered a realistic steady state. Thus, the learning adjustment mechanism model is counter to reality, because students (or athletes) 
can achieve their maximum learning rate for a single examination (or event), but this maximum level is not sustainable over time. The dynamic model discussed subsequently will expand the learning adjustment mechanism to allow for this more realistic possibility.

A second limitation of the simple model in the previous section is that it does not capture the dynamic nature of the learning process. As learning $(k)$ occurs, knowledge $(K)$ grows. Therefore, the model of learning must be altered to accommodate an ever-increasing knowledge base $(K)$, which results in an increasing level of readiness $(R$, equation 5$)$ : students with more knowledge are capable of handling an increasing level of complexity and challenge (Reiser, 2004). Following Obst (1978), the dynamic model of learning can be formulated with the behavioral equation (6). In the dynamic flow model of learning developed subsequently, we will be interested not only in the level or rate of learning $(k)$, but also the rate of change of learning, $k^{\prime}$, where $k^{\prime}=\partial k / \partial t$. Note that the behavioral equation presented in equation (6) is formulated to address the limitations of the simple model in equation (3). We will demonstrate that the dynamic learning adjustment mechanism presented in equation (6) is consistent with economic theory, psychological theory of motivation, and practical classroom experience.

$$
k^{\prime}=-\beta[\Phi-S]
$$

To explore how the system behaves over time, the rates of growth of the variables in equation (5) are found through differentiation, as in equation (7).

$$
(\partial \Phi / \partial t) / \Phi=(\partial a / \partial t) / a+(\partial K / \partial t) / K+(\partial M / \partial t) / M-(\partial C / \partial t) / C
$$

By defining $\Phi^{\prime}$ as the time derivative of $\Phi(=\partial \Phi / \partial t)$, and defining the percentage rate of growth of each variable by the corresponding lowercase letters $(k, m$, and $c)$, we can rewrite equation (7) as equation (8), and equation (6) is replicated as equation (9), where $a^{\prime}=0$, because $a$ is a constant.

$$
\begin{aligned}
\Phi^{\prime} & =(k+m-c) \Phi \\
k^{\prime} & =-\beta[\Phi-S]
\end{aligned}
$$

Equations (8) and (9) complete a system of differential equations that can be solved for the time paths of $\Phi$ and $k$, given a constant (exogenous) rate of challenge, equal to $c$ (Chiang, 1984; Obst, 1978). The system is shown in Figure 3, where the vertical demarcation line represents $\Phi^{\prime}=0$, and the horizontal demarcation line represents $k^{\prime}=0$. The horizontal demarcation line is found by setting $k^{\prime}=-\beta[\Phi-S]=0$. Because $\beta$ is a positive constant, the equality holds when $\Phi=S$. The vertical demarcation line is found by setting $\Phi^{\prime}=(k+m-c) \Phi=0$. Because $\Phi>0$, this equality holds only when $k+m-$ $c=0$, or $k=c-m$, as illustrated in Figure 3. When the learning rate is positive $(k>0)$, it implies that $c>m$. The dynamic system shown in Figure 3 is a vortex 


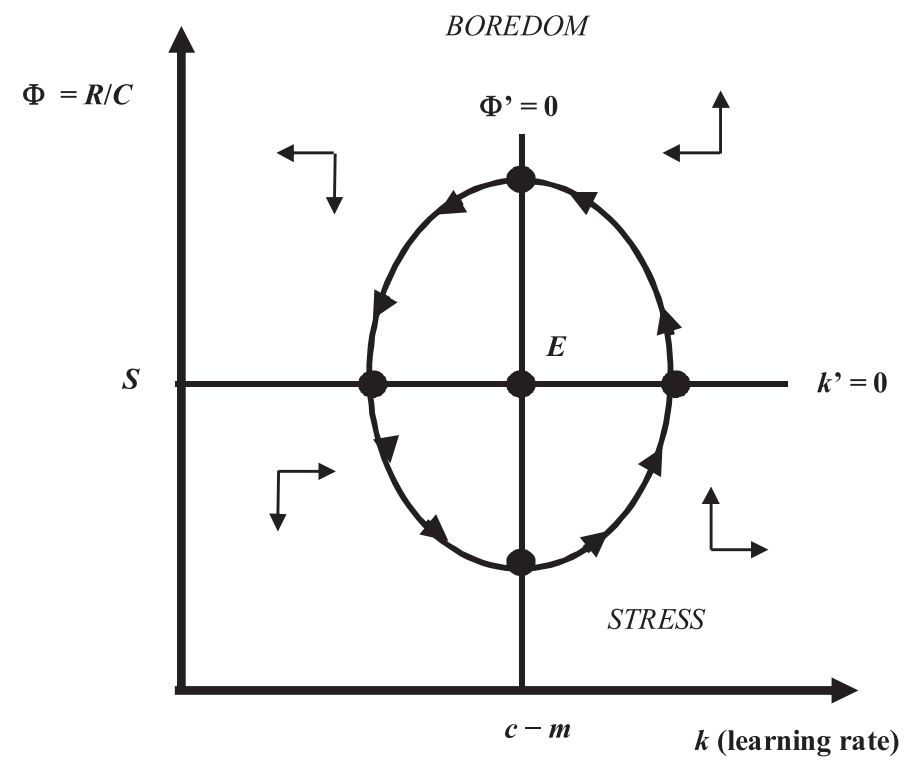

Figure 3. The Dynamics of Learning: A Phase Diagram of the Dynamic Flow Model (Note: This phase diagram maps the dynamic path of a system of differential equations where the determinant of the Jacobian, $\left|J_{E}\right|$, is positive and trace, $\operatorname{tr}\left(J_{E}\right)$, zero. See Appendix for mathematical treatment.)

of unending cycles of stress and boredom, and the steady-state equilibrium $(E)$ is never reached (see Appendix).

Following Obst (1978), it is shown in the Appendix that this result is attributable to the exogeneity of the rate of challenge $(c)$. If instructors targeted the rate of challenge to the learning rate, a different equilibrium could be obtained. If teachers slowed the rate of challenge when the learning rate was so high that undue stress occurred or increased the rate of challenge when boredom occurred, the model could be altered to include an endogenous rate of challenge, as in equation (10).

$$
c=c(k), c^{\prime}(k)<0
$$

This would change equation (8) to incorporate the impact of the learning rate on $c$, as in equation (11).

$$
\Phi^{\prime}=[k+m-c(k)] \Phi
$$

In the modified model, equilibrium occurs when $\Phi^{\prime}=0$, or when $k=c(k)-m$. The Appendix demonstrates that this modified model results in a new equilibrium but remains a vortex, as in the original model, and a steady-state equilibrium would never be reached.

However, a sustainable equilibrium can exist: if the rate of challenge $(c)$ is targeted not to the level of the learning rate $(k)$, but rather to the rate of change 


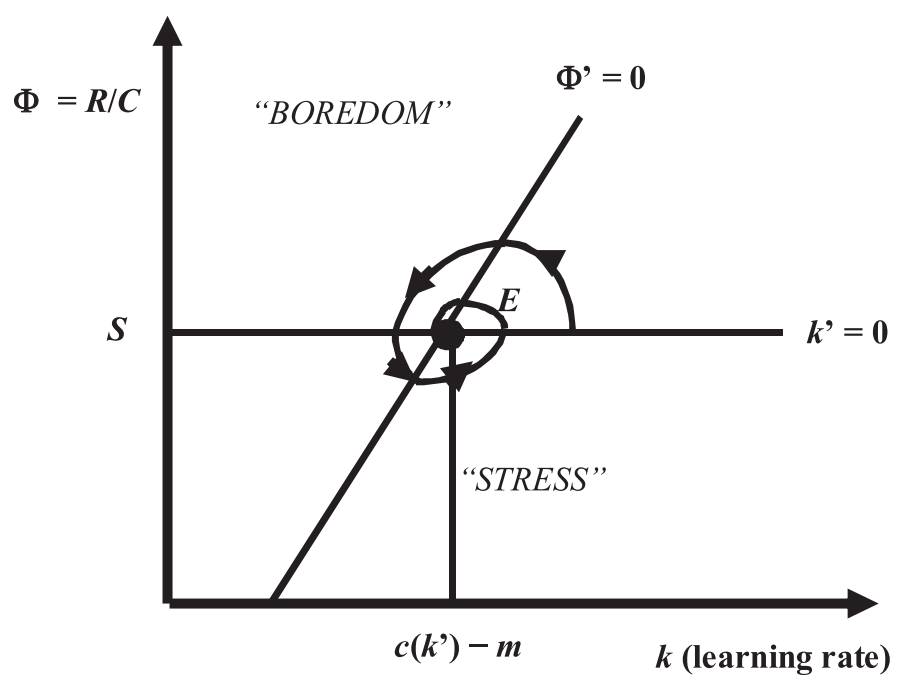

Figure 4. Dynamic Time Path of the Flow Model of Learning (Note: This phase diagram maps the dynamic path of a system of differential equations where the Jacobian has a determinant, $\left|J_{E}\right|$, that is positive and is $\operatorname{trace}, \operatorname{tr}\left(J_{E}\right)$, negative. Under these conditions, the dynamic path is one that leads to a stable equilibrium [Chiang, 1984]. See Appendix for mathematical treatment.)

of learning rate $(k ')$. In this case, a steady-state equilibrium is possible, and the more responsive teachers are to changes in the rate of learning, the more rapidly the dynamic system approaches the steady-state equilibrium. It should be emphasized that the level of learning $(k)$ remains independent of the instructor's change in the rate of challenge. Therefore, students could be working very hard at a high level of learning, or working hard at a low level of learning (for example, if the assignment was not clearly defined or explained). To capture this possibility in the mathematical model, we make the rate of challenge endogenous and a function of the rate of change in the learning rate.

$$
c=c\left(k^{\prime}\right), c^{\prime}\left(k^{\prime}\right)<0
$$

This modification alters equation (8) to equation (13).

$$
\Phi^{\prime}=\left[k+m-c\left(k^{\prime}\right)\right] \Phi
$$

In this version of the model, equilibrium $\left(\Phi^{\prime}=k^{\prime}=0\right)$ occurs when $k=c\left(k^{\prime}\right)$ $-m$. The Appendix shows that in this version of the model, the demarcation line $\Phi^{\prime}=0$ is upward sloping, as in Figure 4, resulting in streamlines that spiral toward the steady-state equilibrium at $\left(\Phi_{e}, k_{e}\right)=\left[S, c\left(k^{\prime}\right)-m\right]$. Thus, the instructional strategy is capable of changing the cyclicality of the vortex into a stable, increasing learning experience where anxiety motivates but does not reach the point of overwhelming students. 
The model results parallel the earlier discussion of Csikszentmihalyi’s (1990, 2003) flow theory of motivation. Using the learning adjustment mechanism presented in equation (8), we have shown mathematically that a learning pathway where challenge $(C)$ and student readiness $(R)$ are balanced is achievable if the rate of challenge $(c)$ is an endogenous variable, calibrated by adjusting the rate of change based on the learning rate ( $\left.k^{\prime}\right)$. Intuitively, the model results imply that teachers who pay careful attention to changes in student learning rates could diminish unproductive outcomes by rapidly modifying the rate of challenge to match the rate of learning. This strategy would result in flow learning experiences by students, as their preparedness is matched by classroom challenge and they master increasingly difficult tasks. The motivation from this flow experience becomes another factor encouraging the steady accumulation of knowledge.

The major outcome of the model that the endogenous rate of challenge provides more efficient learning outcomes is reflected in recent research in educational psychology. Kirschner et al. (2006) reported empirical evidence that minimal guidance during instruction is less effective and efficient than guidance specifically designed to support the cognition processing necessary for learning. Moreno (2004) stated that research-based evidence shows that students learn more from strongly guided learning than from discovery. Thus, there is some evidence to suggest that students are likely to learn more from an endogenous rate of change in the challenge level than an exogenous rate of change in the challenge level.

Figure 5 illustrates the main features of the dynamic model and demonstrates the usefulness of the model results. The flow theory of learning of Figure 1 is repeated in the upper left graph, and the corresponding learning cycle of Figure 3 is in the upper right graph. In Figure 5, these two graphs are drawn for the same learning cycle, with the points in the flow theory graph $\left(t_{0}, t_{1}, \ldots\right)$ matching the labeled points of the vortex graph. To see the learning process in a more intuitive manner, the learning rate $(k)$ is drawn as a function of time, in the lower left graph, and total knowledge $(K)$ is drawn with respect to time in the lower right graph. These two graphs are also drawn with corresponding time labels, to match those in the two upper graphs.

The learning process is viewed as a cycle of anxiety and boredom that results in cycles in the rate of learning. The objective of the teacher is to reduce the volatility in the learning cycles, making the learning process more productive and pleasurable (a flow experience), whenever possible. With this objective, the major implication of the model is for the teacher to match the rate of change in challenge, or the level of complexity, with the rate of change in the learning rate. Beneficial changes in the learning environment will occur the more quickly that instructors can identify changes in the learning rate and act on those changes by synchronizing class lectures, assignments, exams, and activities with those changes. Note that this is independent of the exogenously determined learning rate: the overall learning rate and rate of knowledge accumulation are 

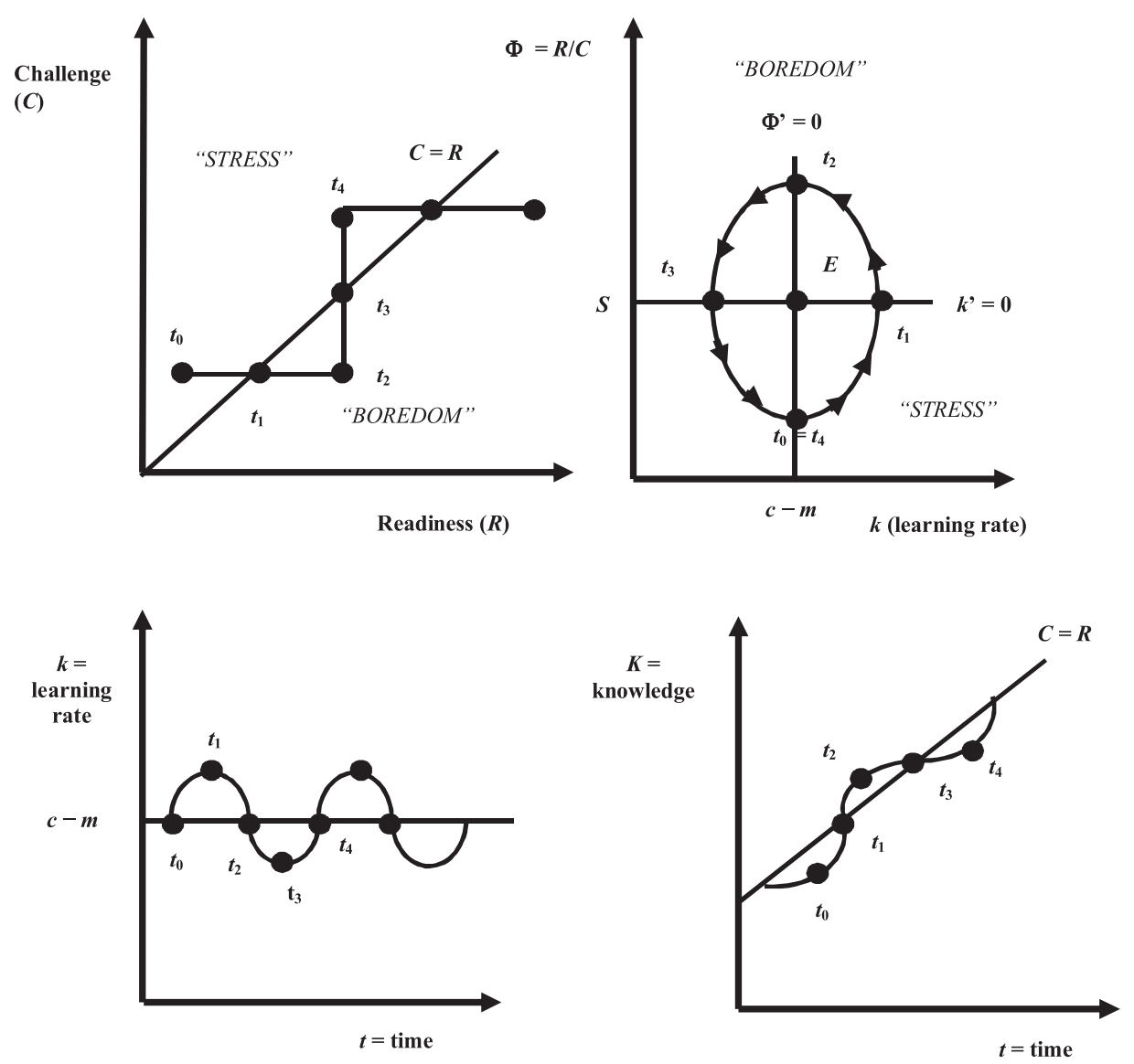

Figure 5. Dynamic Time Path of the Flow Model of Learning (Note: All four graphs correspond to the same learning cycle, with each time $\left[t_{0}, t_{1}, \ldots\right]$ corresponding to the same time and cycle location in all four graphs.)

independent of the learning cycles (Figure 5). Instructors can maintain a set of exogenous, predetermined course learning outcomes while using instructional design and teaching strategies to reduce or eliminate cycles. In this case, the lines in the lower two graphs of Figure 5 remain fixed, while the inefficient cycles around them narrow. This approach allows instructors to determine how well students are doing relative to a predetermined performance level or a set of educational goals determined by the curriculum (Bond, 1996).

Once standards have been determined by the instructor, department, or institution, instructional design and teaching strategies can be used to implement the curriculum (Reiser, 2004). The idea of "backward design," or setting goals prior to choosing instructional methods, was developed by McTighe and Wiggins (2012). This strategy provides a research-based example of how the model 
presented here could be used to encourage and motivate students to achieve predetermined student learning outcomes.

\section{Implications: The Case for Implementing the Dynamic Classroom}

The dynamic model of learning set forth in the previous section shows the ability of instructors to guide students toward flow experience and a steady-state path of knowledge acquisition. The learning adjustment mechanism is consistent with economic behavioral models (Obst, 1978). Furthermore, the concepts built into the model conform to modern understanding of the psychology of motivation and learning (Artino, 2008; Loyens and Gijbels, 2008). Given the theoretical and practical consistency of the model, its outcomes serve as teaching guidelines. Managing the rate of change in the challenge level $(c)$ in the model is key to directing students toward a flow experience and consistent learning. Instructors have different criteria for setting and changing the level of complexity in a course. Model results show that identification of the appropriate rate of change of learning level ( $k$ ') is key to creating an effective learning environment. Ambrose et al. (2010) conclude that "setting challenging but attainable goals is critical for optimally motivating students" (p. 85). Past researchers modeling teaching effectiveness have concluded that a universally optimal approach to teaching does not exist (Lima, 1981, Wetzstein, 1988). This is, in no small part, because of the fact that individual students differ greatly by ability, knowledge, and motivation levels. A teacher can implement the dynamic classroom by attempting to perceive and address the individual differences in stress and boredom that occur within a given term. Nilson $(2016$, p. 99) concludes, "Fortunately, effective motivational techniques and effective teaching techniques greatly overlap. Of course, by definition, more motivated students want to learn more, so they achieve more ... better teaching generates more rewarding learning experiences, which beget more motivation to learn. It is not surprising, then, that you motivate students using the same methods and formats that you do to teach them effectively." The present implications suggest that instructor flexibility and ability to respond to specific groups of students is crucial to a successful dynamic classroom. Providing some flexibility to students can result in a sense of control that can lead to expectations of success (Ambrose et al., 2010, p. 89).

The endogenous rate of change in the level of challenge $(c)$ postulated by the model could be useful in a seminar class, where instructors are able to assign and assess student progress frequently. This allows instructors to pay close attention to how well each individual student is learning ( $\left.k^{\prime}\right)$ and provide the necessary information for altering the rate of challenge level to better match student readiness as students progress through the course. For small, applied fieldtype courses, instructors could provide clear instructions for what is required, provide clear and frequent feedback to students, and maintain a close relationship between rate of challenge and student readiness. 
The endogenous rate of change of challenge could also be employed in a required, core course such as intermediate microeconomics, the foundation for subsequent applied economics courses. The education literature provides several instructional strategies to enhance learning in these courses. Lepper et al. (1993) highlight the distinction between strategies that involve altering the "objective" level of challenge presented to the learner with strategies that seek to change the learner's subjective perceptions of the challenge. In a required, foundational course, the subject matter is typically predetermined by faculty at the department level. Based on feedback on the level of student readiness, objective task difficulty can be modified through instructional design strategies such as supplemental education, educational scaffolding, and self-regulated learning, as explained further in what follows.

Supplemental instruction is an academic support system that could include peer advising, the provision of academic assistance outside of class by tutors, peers, instructors, or computers (Blanc et al., 1983). Reiser (2004) defines educational scaffolding as the process by which a teacher or more knowledgeable peer assists a learner, altering the learning task so the learner can solve problems or accomplish tasks that would otherwise be out of reach. Reiser (2004, p. 275) states that "scaffolding entails a delicate negotiation between providing support and continuing to engage learners actively in the process." Thus, educational scaffolding is finding an optimal level of challenge for learners (Lepper et al., 1993). Self-regulated learning involves explicitly coaching students to think about their study processes and to monitor their learning (Glenn, 2010). Zimmerman (1990) recommends self-regulated learning that includes fast, accurate feedback about how students are doing and making students demonstrate that they understand the provided feedback. Explicit instructions on how to succeed at homework, papers, exams, projects, and presentations can help students succeed.

Lepper et al. (1993) report that the best tutors are "both systematically progressive and highly responsive to the learner. In general, their strategy is to increase the task difficulty across problems, one step at a time, but only when they are sure that the student is ready to advance" (p. 84). The authors go on to say that "strategies for manipulating a learner's sense of challenge may focus on perceptions of the task itself, on perceptions of one's own competence, or on a combination of both" (p. 84). Instructors can motivate students in required courses to perceive difficult tasks as achievable and perceive their own sense of readiness by bolstering self-confidence, evolving curiosity, and promoting a feeling of personal control.

The model results also provide strategies for graduate courses. In many cases, intellectual skills may initially be easily acquired but retained and applied to new situations with difficulty (Gagne and Driscoll, 1988). Driscoll (2005) provides a relevant example: "Students who appeared to understand the new statistical analysis procedure when the instructor went over it in class may 
experience problems trying to use it on new sets of data outside of class" (p. 160). This situation can frequently be a result of differences in student readiness, often quantitative experience and knowledge. The model results suggest that an instructor could pay careful attention to student performance early on in a quantitative graduate course. Because the course goals typically require all students to obtain and use quantitative tools at a given level of proficiency, and there is limited in-class time to review and practice prerequisite mathematical concepts and statistics, a math review could be provided prior to the graduate program or before a specific course. Also, mathematical tools could be provided through online videos or handouts. When the rate of student learning slows, teachers can encourage students to enhance their readiness through these out-ofclass tutorials and peer assistance.

The model results, when combined with Csikszentmihalyi's (1975) flow theory of motivation, offer more guidelines on how to manage the level of complexity that is introduced into a course. First, the flow theory of learning implies that higher levels of learning will occur when instructors set high expectations for student achievement. By "raising the bar," teachers can inspire or motivate students to higher rates of learning. In the original model, an exogenous, higher level of challenge shifts the demarcation line $\Phi^{\prime}=0$ to the right, resulting in a continuation of learning cycles, but at a higher rate of learning. Perhaps one of the most overlooked aspects of college teaching is the ability of teachers to demand greater levels of achievement from students. Moreover, when the challenge of a course is congruent with student ability, an optimal learning experience occurs.

Ambrose et al. (2010) provide a useful list of strategies that help instructors achieve high levels of expectations and achievement among students, emphasizing the model outcomes: (1) align objectives, assessments, and instructional strategies with student learning outcomes; (2) identify an appropriate level of challenge; (3) create assignments and assessments that provide the appropriate level of challenge; (4) provide opportunities for early success; (5) articulate and clarify your expectations; (6) use rubrics; (7) provide targeted feedback; (8) be fair and consistent; (9) educate students about the ways we explain success and failure; and (10) describe effective study strategies (pp. 85-88). Implementation of these strategies is likely to move the classroom toward the "dynamic classroom" approach, where learning cycles of stress and boredom are reduced or, in the ideal case, eliminated.

In order to calibrate the rate of change in the challenge $(c)$ in such a way, a teacher must know her students' level of understanding and ability. Lepper et al. (1993) emphasize the challenge of eliciting this type of information: "Of course, it will be difficult, even for experts, to predict exactly how much difficulty a given problem will pose for a particular learner" (p. 84). Time spent early in a course understanding how much students know and how motivated they are to learn more is a productive investment. To the degree possible, this could help a teacher to manage course complexity to provide enough anxiety that students 
feel challenged to progress (Rauh and Seccia, 2006), but that they can realistically master the material and feel the enjoyment of that mastery. Preassessments and diagnostic assignments conducted early in the term can provide important information about student knowledge and motivation (Ambrose et al., 2010, p. 86). This is one of the major outcomes of the model: for instructors to make a strong effort to learn when their students are struggling or coasting. Frequent evaluation and feedback from students throughout the course are essential to maintaining a proper level of $C$. This corresponds to education literature that suggests that frequent feedback can improve student learning outcomes (Frye, 1999).

Student motivation to learn $(M)$ is exogenous to the model. Motivation is defined as the personal investment that an individual has to reaching a desired state or outcome (Maehr and Meyer, 1997). Wieman (2013) asserts that motivation is likely to be the single most important determinant of learning. Student readiness $(R)$ is defined as a function of motivation $(M)$. Therefore, an understanding of $M$ and how it changes $(m)$ is a large part of $R$ and calibrating $C$ relative to $R$, as previously discussed. Institutions (or specific departments) that foster a culture of learning can increase the level of motivation for students. Educational literature offers guidance in how to accomplish this. Nilson (2016), citing Hobson (2001) and Sass (1989), concludes that "people learn more when they are motivated to do so by the inspiration and enthusiasm of their instructors or other people in their lives" (p. 4). Matching challenge rates $(c)$ with learning rates $\left(k^{\prime}\right)$ will eliminate learning cycles, but knowledge acquisition is also strongly influenced by the level of motivation $(M)$. It should be emphasized that students vary enormously in levels of motivation, and their causes for being motivated to learn. The theoretical model presented here suggests that any increase in motivation would enhance the level of knowledge gained.

Ames (1990) and Maehr and Meyer (1997) provide comprehensive reviews of how motivation relates to learning. Specific strategies that provide a classroom environment conducive to enhancing motivation include (1) connecting the material to the students' interests; (2) providing authentic, real-world tasks; (3) sharing relevance to students' current academic lives; (4) demonstrating the relevance of higher-level skills to students' future professional lives; (5) identifying and rewarding what you value; and (6) sharing your own passion and enthusiasm for the discipline (Ambrose et al., 2010, pp. 83-85; Pintrich, 2003; Wieman, 2013).

\section{Conclusions and Implications for Future Research}

This research provides a mathematical model of the proposition that a stable and sustainable rate of learning occurs when student readiness and challenges are congruent. The model is a dynamic system of simultaneous differential equations, which provides timely, relevant, and important implications for 
teachers and learners. The objective of this research is to utilize economic theory, psychology of motivation, and educational research to construct a mathematical model of learning, in order to identify the major determinants of a successful learning environment for college-level students. Model results demonstrate that instructors who engage students to understand their level of ability and goals to design course expectations build such environments. Instructors willing to place high demands on students (a high level of learning) that are still realistic and flexible relative to student ability (the rate of challenge) offer students an opportunity of a flow learning experience that builds knowledge. Specifically, teachers who target the rate of student learning (rather than simply target the level or rate of knowledge acquisition) may be able to create a learning environment that is productive and enjoyable.

Students differ greatly in readiness, acceptability to challenge, ability, knowledge, motivation, and learning outcomes. Therefore, instructors who adopt a dynamic classroom approach based on the theoretical model will need to pay careful attention, to the degree possible, to each student's learning rate. Nilson (2016) warns, however, “Just don't expect to motivate every one of your students, no matter what you do ... Celebrate those you inspire" (p. 103).

Another contribution of this research is expanding the use of economic models in education. There has been a rich history of economic modeling to evaluate student learning outcomes and differentiate between teaching methods. As fruitful as these ex post analyses have been, there has been a lack of application of conceptual economic models to the process of learning. By adapting Obst's (1978) behavioral model to learning, we demonstrate the appropriateness and effectiveness of economic models in this area and provide a base for future research.

The theoretical model not only yields strategies that enhance student learning, but also suggests how future research could enhance our knowledge of how students learn and how to maximize student learning. One limitation of the current dynamic model of student learning presented previously is the treatment of motivation $(M)$ and knowledge $(K)$ as exogenous variables. Future modeling efforts could explore endogenous levels of motivation and knowledge, allowing for new results and implications. The model could also be expanded by incorporating differences across individual students. We believe that these model extensions are likely to produce important implications for teaching and learning.

The dynamic model also provides important empirical hypotheses. Research that investigates how teachers and students respond to changes in the rate of challenge $(c)$ and compares these to changes in the challenge level $(C)$ would provide evidence for the hypotheses of the model. Specifically, the model puts forth the idea that teachers who focus on reducing fluctuations of boredom and stress by matching the rate of challenge $(c)$ to changes in the rate of student learning $(k$ ') can find a steady-state equilibrium, whereas teachers who target the level of learning $(k)$ will be subjected to continuing volatility of learning 
cycles. These cycles of stress and boredom are costly to students, teachers, and society at large, because they lower the rate of knowledge acquisition. Any strategy, teaching tool, or course improvement that reduces or eliminates these unproductive cycles will result in greater learning levels and a more productive learning environment.

\section{References}

Ambrose, S., M. Bridges, M. DiPietro, M. Lovett, and M. Norman. How Learning Works: Seven Research-Based Principles for Smart Teaching. San Francisco, CA: Wiley and Sons, 2010.

Ames, C.A. “Motivation: What Teachers Need to Know.” Teachers College Record 91 (January 1990):409-21.

Apter, M.J. Reversal Theory: Motivation, Emotion and Personality. London: Routledge, 1989.

Artino, A.R. "Cognitive Load Theory and the Role of Learner Experience: An Abbreviated Review for Educational Practitioners." Association for the Advancement of Computing in Education Journal 16(October 2008):425-39.

Bacdayan, A. "A Mathematical Analysis of the Learning Production Process and a Model for Determining What Matters in Education." Economics of Education Review 16(February 1997):25-37.

Barr, R.B., and J. Tagg. "From Teaching to Learning: A New Paradigm for Undergraduate Education." Change 27(November 1995):12-25.

Baumol, W.J., and W.E. Becker. "The Economist's Approaches.” Assessing Educational Practices: The Contribution of Economics. W.E. Becker and W.J. Baumol, eds. Cambridge, MA: MIT Press, 1995, pp. 1-15.

Becker, W.E. "Economic Education Research: Part II, New Directions in Theoretical Model Building." Journal of Economic Education 14(June 1983a):4-10.

. "Economic Education Research: Part III, Statistical Estimation Methods." Journal of Economic Education 14(June 1983b):4-15.

—. "Quantitative Research on Teaching Methods in Tertiary Education." The Scholarship of Teaching and Learning in Higher Education. W.E. Becker and M.L. Andrews, eds. Bloomington: Indiana University Press, 2004, pp. 265-309.

Becker, W.E., and W.J. Baumol. Assessing Educational Practices: The Contribution of Economics. Cambridge, MA: MIT Press, 1995.

Blanc, R.A., L.E. DeBuhr, and D.C. Martin. "Breaking the Attrition Cycle: The Effects of Supplemental Instruction on Undergraduate Performance and Attrition." Journal of Higher Education 54(January 1983):80-90.

Bond, L.A. "Norm- and Criterion-Referenced Testing." Practical Assessment, Research \& Evaluation 5(November 1996):1-3.

Bowden, E.M., M. Jung-Beeman, J. Fleck, and J. Kounios. "New Approaches to Demystifying Insight." Trends in Cognitive Sciences 9(July 2005):322-28.

Brookfield, S.D. The Skillful Teacher: On Technique, Trust, and Responsiveness in the Classroom. 3rd ed. San Francisco, CA: Jossey-Bass, 2015.

Charkins, R.J., D.M. O’Toole, and J.N. Wetzel. "Linking Teacher and Student Learning Styles with Student Achievement and Attitudes." Journal of Economic Education 16(April 1985):111-20. 
Chiang, A.C. "Simultaneous Differential Equations and Difference Equations." Fundamental Methods of Mathematical Economics. 3rd ed. New York: McGraw-Hill, 1984, pp. 63546.

Csikszentmihalyi, M. Beyond Boredom and Anxiety. San Francisco, CA: Jossey-Bass, 1975.

- Flow: The Psychology of Optimal Experience. New York: Harper and Row, 1990. . Good Business: Leadership, Flow, and the Making of Meaning. New York: Viking, 2003.

Dahlgren, R.A. “Teaching Innovations in Agricultural Economics: An Economic Approach.” American Journal of Agricultural Economics 72(November 1990):873-82.

Driscoll, M.P. Psychology of Learning for Instruction. Needham Heights, MA: Allyn and Bacon, 2005.

Everett, M.W., and M.R. Raven. "A Case Study of Flow Theory in Pre-Service Undergraduate Agriculture, Food, and Natural Resources Education Students." NACTA Journal 59(June 2015):144-48.

Flyer, F., and S. Rosen. "Some Economics of Precollege Teaching." Assessing Educational Practices: The Contribution of Economics. W.E. Becker and W.J. Baumol, eds. Cambridge, MA: MIT Press, 1996, pp. 17-40.

Frye, R. “Assessment, Accountability, and Student Learning Outcomes.” Dialogue 2(February 1999):1-12.

Gagne, R.M., and M.P. Driscoll. Essentials of Teaching. Englewood Cliffs, NJ: Prentice-Hall, 1988.

Glenn, D. "How Students Can Improve by Studying Themselves." Chronicle of Higher Education 56(February 7, 2010):22.

Hebb, D.O. "Drives and the C.N.S. (Conceptual Nervous System).”; Psychological Review 62(July 1955):243-54.

Hobson, E.H. "Assessing Students' Motivation to Learn in Large Classes.” American Journal of Pharmaceutical Education 65(2001):82S.

Kirschner, P.A., J. Sweller, and R.E. Clark. "Why Minimal Guidance During Instruction Does Not Work: An Analysis of the Failure of Constructivist, Discovery, ProblemBased, Experiential, and Inquiry-Based Teaching." Educational Psychologist 41(June 2006):75-86.

Lepper, M.R., M. Woolverton, D.L. Mumme, and J. Gurtner. "Motivational Techniques of Expert Human Tutors: Lessons for the Design of Computer Based Tutors." Computers as Cognitive Tools. S.P. Lajoie and S.J. Derry, eds. Hillsdale, NJ: Lawrence Erlbaum, 1993, pp. 75-105.

Lima, A. "An Economic Model of Teaching Effectiveness." American Economic Review 71(December 1981):1056-59.

Loyens, S., and Gijbels, D. "Understanding the Effects of Constructivist Learning Environments: Introducing a Multi-Directional Approach.” Instructional Science 36(September 2008):351-57.

Maehr, M.L., and H.A. Meyer. "Understanding Motivation and Schooling: Where We've Been, Where We Are, and Where We Need to Go." Educational Psychology Review 9(December 1997):371-409.

Manahan, J. “An Educational Production Function for Principles of Economics.” Journal of Economic Education 14(March 1983):11-16.

McTighe, J, and G. Wiggins. Understanding by Design Framework. Alexandria, VA: Association for Supervision and Curriculum Development, 2012.

Minorsky, N. Nonlinear Oscillations. Princeton, NJ: D. Van Nostrand, 1962. 
Monk, D.H. "The Educational Production Function: Its Evolving Role in Policy Analysis." Educational Evaluation and Policy Analysis 11(1989):31-45.

Moreno, R. "Decreasing Cognitive Load for Novice Students: Effects of Explanatory versus Corrective Feedback in Discovery-Based Multimedia." Instructional Science 32(January 2004):99-113.

Nilson, L.B. Teaching at Its Best: A Research-Based Resource for College Instructors. 4th ed. San Francisco, CA: Jossey-Bass, 2016.

Obst, N.P. “Stabilization Policy with an Inflation-Adjustment Mechanism.” Quarterly Journal of Economics 92(May 1978):355-59.

Pintrich, P. "A Motivational Science Perspective on the Role of Student Motivation in Learning and Teaching Contexts.” Journal of Educational Psychology 95(December 2003):66786.

Rauh, M.T., and G. Seccia. "Anxiety and Performance: An Endogenous Learning-by-Doing Model.” International Economic Review 47(May 2006):583-609.

Reiser, B.J. "Scaffolding Complex Learning: The Mechanisms of Structuring and Problematizing Student Work.” Journal of the Learning Sciences 13(July 2004):273304.

Sass, E.J. "Motivation in the College Classroom: What Students Tell Us." Teaching of Psychology 16(1989):86-88.

Shernoff, D.J., M. Csikszentmihalyi, B. Schneider, and E.S. Shernoff. "Student Engagement in High School Classrooms from the Perspective of Flow Theory." School Psychology Quarterly 18(January 2003):158-76.

Smith, M.K. "Learning Theory.” Encyclopedia of Informal Education. 1999. Internet site: http://www.infed.org/biblio/b-learn.htm (Accessed July 30, 2009).

Stage, F.K., P.A. Muller, J. Kinzie, and A. Simmons. Creating Learning Centered Classrooms: What Does Learning Theory Have to Say? ASHE-ERIC Higher Education Report, Vol. 26, No. 4. Digest. Washington, DC: George Washington University, Graduate School of Education and Human Development, 1998.

Wetzstein, M.E. “An Organonic and Modern Problems Approach for Teaching Agricultural Economics Principles." American Journal of Agricultural Economics 70(February 1988):63-68.

Wetzstein, M.E., and J.M. Broder. "The Economics of Effective Teaching." Journal of Economic Education 16(January 1985):52-59.

Wieman, C. Motivating Learning. Carl Wieman Science Education Initiative at the University of British Columbia, 2013. Internet site: http://www.cwsei.ubs.ca/resources/ files/Motivating-Learning_CWSEI.pdf (Accessed October 15, 2017).

Zimmerman, B.J. "Self-Regulated Learning and Academic Achievement: An Overview." Educational Psychologist 25(January 1990):3-17.

\section{Appendix: Local Stability Analysis of Dynamic Model of Learning}

The dynamic model of learning is represented by equations (8) and (9) in the text, rewritten here as equations $(\mathrm{A}-1)$ and $(\mathrm{A}-2)$.

$$
\begin{aligned}
& \Phi^{\prime}=(k+m-c) \Phi \\
& k^{\prime}=-\beta[\Phi-S]
\end{aligned}
$$


It is assumed that $c$, the rate of change in the level of challenge $\left(c=C^{\prime} / C\right)$ is exogenous. Intuitively, this refers to the extreme case when the instructor is completely disconnected from the class and the students. In this case, the equilibrium of the model occurs at $E=\left(k_{e}, \Phi_{e}\right)=(c-m, S)$. The Jacobian matrix at the equilibrium $\left(J_{E}\right)$ is found in equation $(\mathrm{A}-3)$.

$$
J_{E}=\left(\begin{array}{cc}
\partial k^{\prime} / \partial k & \partial k^{\prime} / \partial \Phi \\
\partial \Phi^{\prime} / \partial k & \partial \Phi^{\prime} / \partial \Phi
\end{array}\right)=\left(\begin{array}{cc}
0 & -\beta \\
\Phi & k+m
\end{array}\right)=\left(\begin{array}{cc}
0 & -\beta \\
S & 0
\end{array}\right)
$$

The local stability of a system of dynamic equations depends on (1) the determinant of $J_{E}$ and (2) the trace of $J_{E}$, both evaluated at the equilibrium (Chiang, 1984; Minorsky, 1962). From (A-3):

$$
\begin{aligned}
\left|J_{E}\right| & =\beta S>0, \\
\operatorname{tr}\left(J_{E}\right) & =0 .
\end{aligned}
$$

Thus, the Jacobian of this system of dynamic equations, evaluated at equilibrium, has trace zero and determinant positive, implying cycles without convergence. Local stability analysis results indicate that this equilibrium is a vortex, with streamlines in the phase diagram (see Figure 3) that form a family of concentric loops, which never reach the equilibrium point. Equilibrium is unattainable for the dynamic system of equations in (A-1) and (A-2).

If the model presented in equations (A-1) and (A-2) is modified to reflect a more realistic instructional environment, where the instructor reacts to student learning rates, we can model a second dynamic system that can be analyzed using local stability analysis for the presence of a stable equilibrium. If instructors alter the rate of challenge $(c)$, based on the rate of learning $(k)$ in a countercyclical fashion, to ameliorate stress and boredom, we achieve

$$
c=c(k), \text { where } c^{\prime}(k)<0 .
$$

By endogenizing the level of challenge, the dynamic system in (A-1) and (A-2) becomes

$$
\begin{aligned}
& \Phi^{\prime}=[k+m-c(k)] \Phi, \\
& k^{\prime}=-\beta[\Phi-S] .
\end{aligned}
$$

The equilibrium of this system is $E=\left(k_{e}, \Phi_{e}\right)=[c(k)-m, S]$. The demarcation line $\Phi^{\prime}=0$ remains vertical in this case, with a different (larger) horizontal intercept, equal to $k=c(k)-m$. The result is a vortex once again, because $\partial \Phi^{\prime} / \partial k=[1-$ $\left.c^{\prime}(k)\right] \Phi$.

The contribution of Obst (1978) was to suggest that monetary policy should target the rate of change of the rate of inflation, rather than the rate of inflation. In our context, instructors could usefully consider gearing changes in the rate of challenge $(c)$ to the rate of change in the rate of learning ( $k$ '). This alternative teaching rule is made explicit in equation (A-9).

$$
c=c^{\prime}\left(k^{\prime}\right), \text { where } c^{\prime}\left(k^{\prime}\right)<0
$$


If the instructor follows this teaching rule, the level of challenge is endogenous and depends on the rate of acceleration or deceleration of learning ( $k$ '). The learning model system of equations becomes (A-10) and (A-11).

$$
\begin{aligned}
\Phi^{\prime} & =\left[k+m-c\left(k^{\prime}\right)\right] \Phi \\
k^{\prime} & =-\beta[\Phi-S]
\end{aligned}
$$

In this modified model, $\Phi^{\prime}=0$ implies that, in equilibrium, $k=c\left(k^{\prime}\right)-m$. Additionally, the equilibrium condition, $k^{\prime}=0$, implies that $\Phi=S$ in equilibrium, defined as $\Phi^{\prime}=k^{\prime}=0$. The Jacobian of the modified model evaluated at equilibrium is equal to

$J_{E}=\left(\begin{array}{cc}\partial k^{\prime} / \partial k & \partial k^{\prime} / \partial \Phi \\ \partial \Phi^{\prime} / \partial k & \partial \Phi^{\prime} / \partial \Phi\end{array}\right)=\left(\begin{array}{cc}0 & -\beta \\ \Phi & k+m-c\left(k^{\prime}\right)-c^{\prime}\left(k^{\prime}\right)(-\beta)\end{array}\right)=\left(\begin{array}{cc}0 & -\beta \\ S & -c^{\prime}(0)(-\beta)\end{array}\right)$

We know that $c^{\prime}(0)<0$, from the assumption that $c^{\prime}\left(k^{\prime}\right)<0$. The determinant and trace are

$$
\begin{aligned}
\left|J_{E}\right| & =\beta S>0, \\
\operatorname{tr}\left(J_{E}\right) & =-c^{\prime}(0)(-\beta)<0 .
\end{aligned}
$$

The Jacobian at equilibrium is trace negative and determinant positive, which corresponds to either (1) a stable focus or (2) a stable node. Whether a stable focus or node occurs depends on the magnitude of $\left(\operatorname{tr} J_{E}\right)^{2}$ and $4\left|J_{E}\right|$ : if $\left(\operatorname{tr} J_{E}\right)^{2}>4\left|J_{E}\right|$, a stable node occurs, whereas if $\left(\operatorname{tr} J_{E}\right)^{2}<4\left|J_{E}\right|$, a stable focus occurs. The larger the absolute value of the derivative $c^{\prime}(0)$, the larger the value of $\operatorname{tr} J_{E}$, and the more probable $\left(\operatorname{tr} J_{E}\right)^{2}$ $>4\left|J_{E}\right|$, the condition necessary for the existence of a stable node, with streamlines flowing noncyclically toward the node (Figure 4).

Intuitively, this result suggests that the more responsive an instructor is in matching the rate of challenge with the rate of change in the learning rate, the more likely a stable equilibrium is reached, and the more rapidly the model reaches the steady-state equilibrium. Graphically, this can be seen in Figure 4: a larger derivative $c^{\prime}(k$ ') results in a flatter demarcation line $\Phi^{\prime}=0$. The closer to horizontal the demarcation line, the faster the streamlines cycle toward equilibrium. Therefore, the more perceptive an instructor is to changes in learning rates, and the more rapidly the instructor can alter the rate of challenge, the faster a steady-state learning environment can be reached, and the cyclicality of stress and boredom can be reduced or brought to a halt. 